\title{
Glass transition in Ultrathin Polymer Films : A Thermal Expansion Study
}

\author{
M. Bhattacharya, M. K. Sanyal \\ Surface Physics Division, Saha Institute of Nuclear Physics, \\ 1/AF, Bidhannagar, Kolkata 700 064, India. \\ Th. Geue, U. Pietsch \\ Institut für Physik, Universität Potsdam, \\ D-14469 Potsdam, Germany.
}

June 26, 2018

\begin{abstract}
Glass transition process gets affected in ultrathin films having thickness comparable to the size of the molecules. We observe systematic broadening of glass transition temperature $\left(T_{g}\right)$ as the thickness of the polymer film reduces below the radius of gyration but the change in the average $T_{g}$ was found to be very small. Existence of reversible negative and positive thermal expansion below and above $T_{g}$ increased the sensitivity of our thickness measurements performed using energy dispersive x-ray reflectivity. A simple model of $T_{g}$ variation as a function of depth expected from sliding motion could explain the results. We observe clear glass transition even for $4 \mathrm{~nm}$ polystyrene film that was predicted to be absent from ellipsometry measurements of thicker films.
\end{abstract}

\section{Introduction}

The phase transition process of non-crystalline materials from a glassy to molten state has remained an outstanding problem in condensed matter physics especially when these materials are confined in nanometer length 
scale. This is a subject of intense research for macromolecules like polymers as the confinement effect becomes prominent even in films having thickness of tens of nanometers. The effect of nano-confinement on the glass transition temperature $\left(T_{g}\right)$ of ultrathin polymer films is being studied extensively [1, 2] as the results may influence our basic understanding about glass transition mechanism in general [3, 4] and dynamics of polymer chains near glass-melt transition, in particular [5, 6]. These studies are also important for technological applications of nanometer-thick polymer films [7. In most of these investigations the thicknesses of polymer films are measured using ellipsometry technique [8] as a function of temperature across glass-melt transition. The $T_{g}$ in these measurements was defined [2, 8] using temperatures at which thermal expansion deviates from linearity above $\left(T_{+}\right)$and below $\left(T_{-}\right)$the glass transition with $T_{g}=0.5\left(T_{+}+T_{-}\right)$. In ellipsometry measurements, $T_{+}$ were found to be constant and $T_{-}$exhibited rapid reduction as the thicknesses of the polymer films approached $10 \mathrm{~nm}$ - a thickness below which $T_{g}$ could not be determined from thickness measurements due to contrast problem [2, 3, 8]. As $T_{+}$was constant, both broadening of glass transition $\Delta T$ (= $T_{+}-T_{-}$) and $T_{g}$ itself were found to follow the variation of $T_{-}$with thickness in polystyrene thin films. It was assumed that for thin polymer films a top layer of thickness around $5 \mathrm{~nm}$ is in molten state even at room temperature. The effect of the molten top free surface on reduction of $T_{g}$ has been confirmed by capping the polymer films by a metal layer [9]. The measurements were performed on primarily polystyrene films having thickness between 10 to 100 nanometer and the values of $T_{g}$ thus obtained from various different measurements could be parameterized with an empirical relation

$$
T_{g}^{f}=T_{g}^{b}\left[1-(A / h)^{\delta}\right]
$$

where $T_{g}^{b}$ and $T_{g}^{f}$ are glass transition temperatures for bulk polymer and for a film of thickness $h$ respectively [2, 3, 8]. This equation obviously indicates finite $T_{g}$ above a thickness $\mathrm{A}$ and its value was found to be $3.2 \mathrm{~nm}$. It is indeed very interesting to note that except one data set 4], large number of measurements [2, 8, 9] performed in different experimental conditions with variety of surface treatments of silicon substrates, which were used for depositing polystyrene films of various molecular weights, followed this empirical relation with $\mathrm{A}=3.2 \mathrm{~nm}$ and $\delta=1.8$. The implication of this intriguing result is that a film of $3.2 \mathrm{~nm}$ will not show finite $T_{g}$ and even for a $8 \mathrm{~nm}$ film $T_{g}^{f}$ will be below room temperature. Our results however exhibit evidence of glass transition for films having even $4 \mathrm{~nm}$ thickness and the obtained value of 
$T_{g}^{f}$ remains close to $T_{g}^{b}$ though transition becomes broad as the thickness is reduced. We also demonstrate that one has to use the concept of distributed glass transition temperature [6] to explain the dramatic increase in $\Delta T$ as the thickness becomes comparable to the radius of gyration $\left(R_{g}\right)$ of the polymer. In a recent calorimetric measurement, ultrathin (1 to 10nm) polymer films exhibited [10 pronounced glass transition temperature and this measurement also showed minor change in $T_{g}$ with thickness but confirmed the broadening of glass transition with reduction of film thickness, which is in agreement with our findings. Moreover, the results presented here are consistent with the earlier thickness measurement studies using conventional x-ray reflectivity and ellipsometry techniques except for the films less than $20 \mathrm{~nm}$ for which sensitivity of earlier thickness measurements were not satisfactory [2, 3, 8.

\section{Experimental Studies}

\subsection{Sample preparation}

We have studied thermal expansion behaviour of spin coated ultrathin Polystyrene films in the thickness range of 4 to $31 \mathrm{~nm}$. Polystyrene (Aldrich, USA, $M_{w}=212,400$, radius of gyration $R_{g} \sim 12.6 \mathrm{~nm}$ and $T_{g}^{b}=373 \mathrm{~K}$ ) thin films were deposited by spin coating solutions of the polymer in toluene at around $3000 \mathrm{rpm}$ onto hydrophilic silicon (100) substrates prepared following RCA cleaning procedure. In this chemical treatment, substrates were first heated with $30 \%$ ammonium hydroxide $\left(\mathrm{NH}_{4} \mathrm{OH}\right)$ and $30 \%$ hydrogen peroxide $\left(\mathrm{H}_{2} \mathrm{O}_{2}\right)$ solution (1:1 volume ratio) for 10 minutes followed by a rinsing with acetone and alchohol and finally washed with purifies water having 18.2 Mohm cm resistivity (Millipore, USA). The film thickness was controlled by changing the rotation speed and by choosing appropriate concentration of the solution from 1-6.5 mg/ml of toluene.

\subsection{Sample characterization}

It is known that any property of polymer films determined through thickness measurements is sensitive to dewetting phenomena and one has to be careful especially for ultrathin polymer films [5, 11, 12, 13. We present here results of polymer films deposited on hydrophilic silicon (100) substrate, which do not 
show any dewetting even after repeated thermal cycling. The polymer films were heated in a sample cell under vacuum $\left(3 \times 10^{-2}\right.$ mbar $)$ at a temperature (403K) above $T_{g}^{b}$ of PS and we have monitored the top surface of the film with optical microscope. We have performed these measurements for both thick and ultrathin PS films down to $4.3 \mathrm{~nm}$ range reported here. In Fig. 1(a), the obtained ADR profiles and corresponding fits are presented as a function of temperature for a $4.3 \mathrm{~nm}$ thick polymer film. From our analysis, it is seen that the roughness values of this film of $4.3 \mathrm{~nm}$ thickness measured at $303 \mathrm{~K}$ and $403 \mathrm{~K}$ come out to be $2.8 \AA$ and $3.3 \AA$ respectively (Fig. $1(\mathrm{a})$ ) and we could get back same parameter after repeated thermal cycling. Dewetting of film would have increased the roughness drastically [5] and we could not have got back same reflectivity profile after thermal cycling.

We have also performed Energy Dispersive X-ray Spectrometry measurements on chemically treated silicon substrate and the polymer films. In the inset of Fig. 1(a), part of EDX spectra of a substrate and polymer film collected at $5 \mathrm{keV}$ energy in scanning electron microscope are shown to indicate that the film is free from other contaminants and our impression is that clean environment should be used to avoid dewetting of the polystyrene films deposited on hydrophilic silicon substrates.

\subsection{Transverse $\mathrm{x}$-ray diffuse scattering measurements}

We have studied the in-plane correlation present in polymer films by measuring transverse $\mathrm{x}$-ray diffuse scattering as a function of temperature at X22C beamline of National Synchrotron Light Source, Brookhaven National Laboratory. In Fig. 1(b) and (c) we have shown data collected at two temperatures as a function of $q_{x}[=(2 \pi / \lambda)(\cos \beta-\cos \alpha)]$ at three fixed $q_{z}[=(2 \pi / \lambda)(\sin \alpha+\sin \beta)]$ values, where $\alpha$ and $\beta$ are incidence and exit angle of x-rays of wavelength $\lambda$. Capillary waves are of particular importance in case of soft matter interfaces and polymer films are known [11, 13] to exhibit charactaristic of capillary wave correlation [11, 12, 13, 14, 15]. From theory of capillary waves, the height-height correlation function can be expressed as

$$
C(R)=(B / 2) K_{0}(\kappa R),
$$

where $K_{0}(\kappa R)$ is modified Bessel function, $\kappa$ is low wave vector cut off and $B=k_{B} T /(\pi \gamma)$ with $\gamma$ as the surface tension at temperature $\mathrm{T}$ and $k_{B}$ $=1.3806 \times 10^{-23} \mathrm{~J} / \mathrm{K}$ is the Boltzmann constant. Within experimentally 
accessible wave vector range, $K_{0}(\kappa R)$ can be well approximated as $\left[K_{0}(\kappa R) \approx\right.$ $\left.-\ln (\kappa R / 2)-\gamma_{E}\right]$, where $\gamma_{E}$ is the Euler constant. Inserting this type of logarithmic correlation to the scattering function calculation and considering an approximation of the transmission functions $|T(\alpha)|^{2}|T(\beta)|^{2}$ with that of substrate the observed intensity can be obtained as [15],

$$
\begin{aligned}
I= & I_{0} \frac{R\left(q_{z}\right) q_{z}}{2 k_{0} \sin \alpha} \frac{1}{\sqrt{\pi}} \exp \left[-q_{z}^{2} \sigma_{e}^{2}\right] \Gamma\left(\frac{1-\eta}{2}\right) \\
& \times{ }_{1} F_{1}\left(\frac{1-\eta}{2} ; \frac{1}{2} ; \frac{q_{x}^{2} L^{2}}{4 \pi^{2}}\right)|T(\alpha)|^{2}|T(\beta)|^{2}
\end{aligned}
$$

Here ${ }_{1} F_{1}(x ; y ; z)$ is the Kummar function and we could fit all the data using Eq. (3) self consistently [15] (Fig. 1(b) and (c)) with this line shape. From our analysis, the values of $\mathrm{B}$ come out to be $1.1 \AA^{2}$ and $2.1 \AA^{2}$ for 333 $\mathrm{K}$ and $393 \mathrm{~K}$ respectively. Dewetting of films would have generated different line shapes as observed earlier [13. We find that the films retain this line shape through thermal cycling confirming that dewetting is not taking place.

\subsection{Thickness measurements through reflectivity}

Thermal expansion studies of the polymer thin films were carried out in the temperature range of $308 \mathrm{~K}$ to $433 \mathrm{~K}$ using both energy (EDR) and angle (ADR) dispersive x-ray reflectivity techniques [16]. In specular condition, the intensity of reflected x-ray beam is measured as a function of wave vector transfer $q_{z}(=4 \pi E \sin \theta /(h c)$ with $\mathrm{hc}=12.3986 \mathrm{keV} \AA)$ where the incident and exit angle $\theta$ are kept equal. The ADR experiments were performed by changing the angle $\theta$ in a laboratory set up [12] and the EDR measurements were carried out at the EDR beamline [17] of BESSY-II synchrotron (Berlin, Germany) by keeping the angle $\theta$ constant ( $1^{\circ}$ here). All the reflectivity measurements both in angle and energy dispersive mode were carried out keeping the samples in vacuum $\left(10^{-2}\right.$ mbar $)$.

We have already demonstrated [16] that the polymer films deposited on hydrophilic $\mathrm{Si}(100)$ substrate exhibit reversible negative and positive thermal

expansion below and above $T_{g}^{b}$ respectively. Our observation has been confirmed in an independent measurement and predicted large relaxation time has also been measured [18. Negative thermal expansion was also observed in polycarbonate ultrathin film while cooling the films below apparent $T_{g}$ [19]. 
The existence of positive and negative thermal expansion [16, 18, 19, 20] coefficients above and below $T_{g}$ improved the sensitivity of measurement of glass transition temperature. Moreover, we used here the EDR technique, which allowed us to collect large number of reflectivity profiles around $T_{g}^{b}$ in quick succession. EDR technique is ideal method to investigate small thermal expansion in thin polymer films. In Fig. 2 we have shown the measured ADR and EDR profiles collected from a polymer film at room temperature along with the fit giving us a thickness of $4.5 \mathrm{~nm}$. The procedure of EDR data extraction and other experimental details of EDR beamline of BESSYII synchrotron has been described earlier [16, 21]. We have also shown some of the representative EDR profiles at three different temperatures along with the fitted curves in the inset of Fig. 2. The change in thickness is apparent in the shift of minimum of reflectivity curves itself. In the analysis [21] of normalized EDR and ADR data, we have used a model of a polymer film of constant electron density with two roughness profiles at film-air and filmsubstrate interfaces. The obtained thickness as a function of temperature for this film is shown in Fig. 3(a). Initially, the $4.5 \mathrm{~nm}$ PS film showed negative thermal expansion with the coefficient of $-3.7 \times 10^{-3} \mathrm{~K}^{-1}$ over $\sim 30$ K temperature range starting from $308 \mathrm{~K}$. Near 339 K, negative thermal expansion decreases to give rise to a flat region up to $\sim 408 \mathrm{~K}$ in the thickness vs. temperature curve. Above $\sim 408 \mathrm{~K}$, the film thickness increases and the resultant expansion coefficient comes to be $2.5 \times 10^{-3} \mathrm{~K}^{-1}$, much greater than that of bulk PS [16, 21]. The temperatures $T_{+}$and $T_{-}$were determined by fitting two straight lines at high and low temperature region respectively as shown in the Fig. 3(a). The calculated $T_{g}^{f}$ obtained from $0.5\left(T_{+}+T_{-}\right)$ (indicated as $T_{m}$ ) and from the intersection of the two fitted lines as shown in Fig. 3(a) were found to be very close for all the films. In Fig. 3(b) and (c) we have shown results of the films having room temperature thickness of $5.7 \mathrm{~nm}$ and $23.1 \mathrm{~nm}$ respectively. We find that values of $T_{g}^{f}$ remains very close to that for the bulk polymer $T_{g}^{b}$ for all films but broadening of glass transition $\Delta T\left(=T_{+}-T_{-}\right)$reduces as the film thickness increases.

\section{$3 \quad$ Results and Discussions}

It has been argued [6] that for ultrathin films one can expect two types of diffusion processes, the first one is standard motions controlled by the free volume and the second one is sliding (reptation) motions for chains having 
end points at the free surface. One can expect increase in the sliding motions for molecules having centre of mass near surface giving rise to decrease in $T_{g}$ towards free surface of a film. In a recent fluorescence measurement of multilayer polymer films, systematic reduction of $T_{g}$ was observed for the molecules close to free surface [22]. We have used this concept for explaining observed thickness variation with temperature and large broadening $(\Delta T)$ of glass transition. In our simple model, we have assumed that a film is composed of small pseudo-layers of equal thickness having different glass transition temperatures $\left(T_{g}\right)$. Each of these pseudo-layers continues to show negative thermal expansion up to the corresponding $T_{g}$, independent of other pseudo-layers and then expands giving rise to positive thermal expansion. The negative and positive thermal expansion coefficients in each of these pseudo-layers were kept equal to that of the total film. The $T_{g}$ $\mathrm{s}$, obtained from best fit of the measured curve corresponding to the $T_{g}$ of six pseudo-layers used here for the $4.5 \mathrm{~nm}$ and $5.7 \mathrm{~nm}$ PS films, are indicated in the inset of Fig. 3(a) and 3(b). The generated curve by adding all these six pairs of straight line match quite well with experimental data. The fit can be improved by using more number of pseudo-layers but the basic feature is quite clear even in this simple model. In case of $23.1 \mathrm{~nm}$ film (Fig. 3(c)) we used five pseudo-layers instead of six as $\Delta T$ has reduced significantly. The molecules near surface have enhanced probability of sliding motion and thereby lower effective $T_{g}$. Effective $T_{g}$ of pseudo-layers progressively approach bulk value $T_{g}^{b}$ as the position of the pseudo-layer lowers and this value becomes even more than $T_{g}^{b}$ for the pseudo-layer situated near film substrate interface due to strong interaction between polymer and substrate. In our model, $T_{-}$and $T_{+}$are effective $T_{g}$ of the top and bottom pseudo-layers in each film. The calculated values of $T_{g}^{f}, T_{+}$and $T_{-}$for all the films are shown in Fig. 4(a). In Fig. 4(b), we have presented obtained absolute value of thermal expansion $(|\alpha|)$ both below and above $T_{g}$ and the extracted $\Delta T$ for the five PS films of different thicknesses. We note that the values of $|\alpha|$ in these films decreases exponentially as the film thickness is increased and from $\sim 2 R_{g}$-thick PS films, the thermal expansion above $T_{g}$ become close to the volume expansion of bulk PS as observed earlier [16]. The broadening of glass transition $\Delta T$ also exhibit an exponential dependence and we could parameterized all the curves with a function $a_{1}\left[\exp \left(-h / a_{2}\right)\right]+a_{3}$. The values of fitting parameters $a_{1}, a_{2}, a_{3}$ for negative and positive thermal expansion coefficients come out to be $4.1 \times 10^{-2} \mathrm{~K}^{-1}, 1.8 \mathrm{~nm}, 2.2 \times 10^{-4} \mathrm{~K}^{-1}$ and $5.6 \times$ $10^{-3} \mathrm{~K}^{-1}, 4.4 \mathrm{~nm}, 4.5 \times 10^{-4} \mathrm{~K}^{-1}$ respectively. For the $\Delta T$ data, we obtain 
these parameters as $85.46 \mathrm{~K}, 10.8 \mathrm{~nm}$ and $15.1 \mathrm{~K}$ respectively.

In Fig. 4(a) we have also shown variation of $T_{g}^{f}$ with thickness and found that there is a reduction of $7 \mathrm{~K}$ as the thickness of the film is reduced to 4 $\mathrm{nm}$ with an error bar around $3 \mathrm{~K}$. Our results agree with the calorimetric results [10] which indicated no appreciable dependence of glass-transition temperature on film thickness. In fact one averaged value of $T_{g}$ for the ultrathin films having large $\Delta T$ is an ill-defined parameter. Referring back to Eq. (1) and its remarkable success in explaining large number of ellipsometry results, we note that this equation represents essentially variation of $T_{-}$as $T_{+}$was found to be constant in ellipsometry measurements. We extracted the values of $T_{-}$as a function of thickness from the published values [2, 8] of $T_{g}^{f}$ knowing the constant values of $T_{+}$in those ellipsometry measurements. The extracted values of $T_{-}$are shown with the obtained values of $T_{-}$from our measurements in the inset of Fig. 4(a). Excluding the present data, one can indeed get respective values of $A$ and $\delta$ as $3.76 \pm 0.88 \mathrm{~nm}$ and $1.76 \pm$ 0.35 , by fitting (dashed line) the Eq. (1) as reported [2, 3, 8]. For fitting the data set over the entire thickness range shown in inset of Fig. 4(a) with the Eq. (1) one has to ignore the earlier measured ellipsometry data below 20 $\mathrm{nm}$ that had problem of contrast [8]. We obtained the values of $0.47 \pm 0.21$ $\mathrm{nm}$ and $1.13 \pm 0.23$ for $A$ and $\delta$ respectively from this fitting (solid line). It may be noted that the obtained value of $A$ here is close to the segment length of polystyrene $0.67 \mathrm{~nm}$ indicating that polymer film of finite thickness will always have finite $T_{-}$. Moreover increase in $T_{+}$for ultrathin films will keep $T_{g}^{f}$ close to $T_{g}^{b}$.

\section{Conclusion}

In conclusion, we have shown by measuring thickness of ultrathin polystyrene films as a function of temperature that conventional glass transition takes place even for a $4.5 \mathrm{~nm}$ film and we do not observe an appreciable dependence of average $T_{g}$ as a function of thickness. We find large broadening of glass transition temperature occur as the film thickness reduces below radius of gyration. We could explain this broadening by invoking continuous glass transition temperature as a function of depth arising due to variation of the contribution of sliding motion in the diffusion process. Probability of having ends of a polymer chain at the free surface, the essential requirement for sliding motion, increases as the centre of mass of the chain comes closer to 
the free surface [6] giving rise to lowest but finite $T_{g}$, marked as $T_{-}$in a film.

\section{ACKNOWLEDGEMENTS}

The authors are grateful to Manabendra Mukherjee and Yves Bodenthin for valuable discussions. The authors are also thankful to Joydeep Basu and John Hill for their support in NSLS experiment. This work was supported by DST-DAAD India Germany collaboration programme.

\section{References}

[1] G. Reiter and P. G. de Gennes, Eur. Phys. J E 6, 25 (2001).

[2] J. A. Forrest, Eur. Phys. J E 8, 261 (2002); J. A. Forrest and K. DalnokiVeress, Adv. Colloid Interface Sci. 94, 167 (2001) and references there.

[3] J. L. Keddie, R. A. L. Jones, and R. A. Cory, Europhys. Lett. 27, 59 (1994).

[4] W. E. Wallace, J. H van Zanten, and W. L. Wu, Phys. Rev. E 52, R3329 (1995)

[5] G. Reiter, Europhys. Lett. 23, 579 (1993); G. Reiter, Macromolecules 27, 3046 (1994).

[6] P. G. de Gennes, Eur. Phys. J. E 2, 201 (2000).

[7] C. J. Ellison, R. L. Ruszkowski, N. J. Fredin, and J. M. Torkelson, Phys. Rev. Lett. 92, 095702 (2004).

[8] S. Kawana and R. A. L. Jones, Phys Rev. E 63, 021501 (2001); S. Kawana and R. A. L. Jones, Eur. Phys. J. E 10, 223 (2003).

[9] J. S. Sharp and J. A. Forrest, Phys. Rev. Lett. 91, 235701 (2003).

[10] M. Yu. Efremov, E. A. Olson, M. Zhang, Z. Zhang, and L. H. Allen, Phys. Rev. Lett. 91, 085703 (2003).

[11] M. Tolan, O. H. Seeck, J. -P. Schlomka, W. Press, J. Wang, S. K. Sinha, Z. Li, M. H. Rafailovich, and J. Sokolov, Phys. Rev. Lett. 81, 2731 (1998) 
[12] J. K. Basu, S. Hazra, and M. K. Sanyal, Phys. Rev. Lett. 82, 4675 (1999).

[13] W. Zhao, M. H. Rafailovich, J. Sokolov, L. J. Fetters, R. Plano, M. K. Sanyal, S. K. Sinha, and B. B. Sauer, Phys. Rev. Lett. 70, 1453 (1993).

[14] J. Daillant and M. Alba, Rep. Prog. Phys. 63, 1725 (2000).

[15] M. K. Sanyal, S. K. Sinha, K. G. Huang, and B. M. Ocko, Phys. Rev. Lett. 66, 628 (1991).

[16] M. Mukherjee, M. Bhattacharya, M. K. Sanyal, T. Geue, J. Grenzer, and U. Pietsch, Phys. Rev. E 66, 061801 (2002).

[17] Y. Bodenthin, J. Grenzer, R. Lauter, U. Pietsch, P. Lehmann, D. G. Kurth, and H. Möhwald, J. Synchrotron Rad. 9, 206 (2002).

[18] T. Miyazaki, K. Nishida, and T. Kanaya Phys. Rev. E 69, 022801 (2004).

[19] Christopher L. Soles, Jack F. Douglas, Ronald L. Jones, and Wen-li Wu, Macromolecules 37, 2901 (2004).

[20] William J. Orts, John H. van Zanten, Wen-li Wu, and Sushil K. Satija, Phys. Rev. Lett. 71, 867 (1993).

[21] M. Bhattacharya, M. Mukherjee. M. K. Sanyal, Th. Geue, J. Grenzer, and U. Pietsch, J. Appl. Phys. 94, 2882 (2003).

[22] C. J. Ellison and J. M. Torkelson, Nature Mater 2, 695 (2003). 


\section{Figure captions:}

Figure 1: (a) The measured ADR profiles (shifted vertically) of a $4.3 \mathrm{~nm}$ film with fits are shown as a function of temperature, top to bottom, for a thermal cycle. The shift of minima, marked by dashed lines, indicate the reversibility of thermal expansion. In the inset part of the EDX spectra collected from a Silicon substrate (line) and a ultrathin polymer film (line+circle) are shown. In (b) and (c), log-log plots of transverse diffuse scattering intensity are presented along with the fitted curves 15 (solid lines) as a function of $q_{x}$ at three values of $q_{z}$ (with different symbols) at $333 \mathrm{~K}$ and $393 \mathrm{~K}$ respectively. Also the backgrounds used for fitting are indicated by dashed lines.

Figure 2: The ADR (circle) and extracted [16] EDR (square) profiles at room temperature for another $4.5 \mathrm{~nm}$ PS film are shown along with the fitted line. Two dashed lines indicate the usable EDR data range. Typical EDR profiles for this film at temperatures $333 \mathrm{~K}$ (star), $373 \mathrm{~K}$ (up triangle), $429 \mathrm{~K}$ (square) and $321 \mathrm{~K}$ (down triangle) are also shown along with the fitted profiles in the inset.

Figure 3: (a) The obtained thickness variation for the $4.5 \mathrm{~nm}$ PS film is presented as a function of temperature for heating cycle along with the generated curve obtained from our model. The temperatures $T_{-}, T_{+}, T_{m}, T_{g}^{f}$ are indicated and the broadening of glass transition $\Delta T$ is marked by straight dashed lines with arrows. Another straight dashed line is used to show $T_{m}$ and $T_{g}^{f}$ are very close (refer text for details).In the inset of (a) the six straight lines are shown indicating the $T_{g}$-s of six pseudo-layers as obtained from our model (the profiles are vertically shifted for clarity). Results of similar analysis is shown for films having room temperature thickness of $5.7 \mathrm{~nm}$ (b) and $23.1 \mathrm{~nm}$ (c) (refer text for details).

Figure 4: (a) $T_{+}$(solid up triangle), $T_{g}^{f}$ (open circle), and $T_{-}$(solid down triangle) are presented with the error bars as a function of film thickness for five PS films of thicknesses $4.5 \mathrm{~nm}, 5.7 \mathrm{~nm}, 11.6 \mathrm{~nm}, 23.1 \mathrm{~nm}$ and $31.6 \mathrm{~nm}$. In the inset of (a) the solid line represents the fit with the Eq. (1) to the

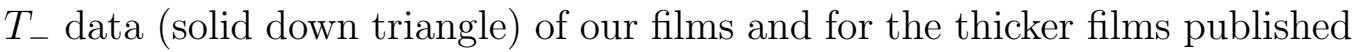
earlier 2]. Inclusion of earlier published thinner film $T_{-}$values (open down triangle) clearly indicate rapid reduction (dashed line) of $T_{-}$erroneously. (b) The obtained negative thermal expansion (solid square) and positive thermal expansion (open square) coefficients together with $\Delta T$ (star) for the five PS films are presented with exponential fits (refer text for details). 


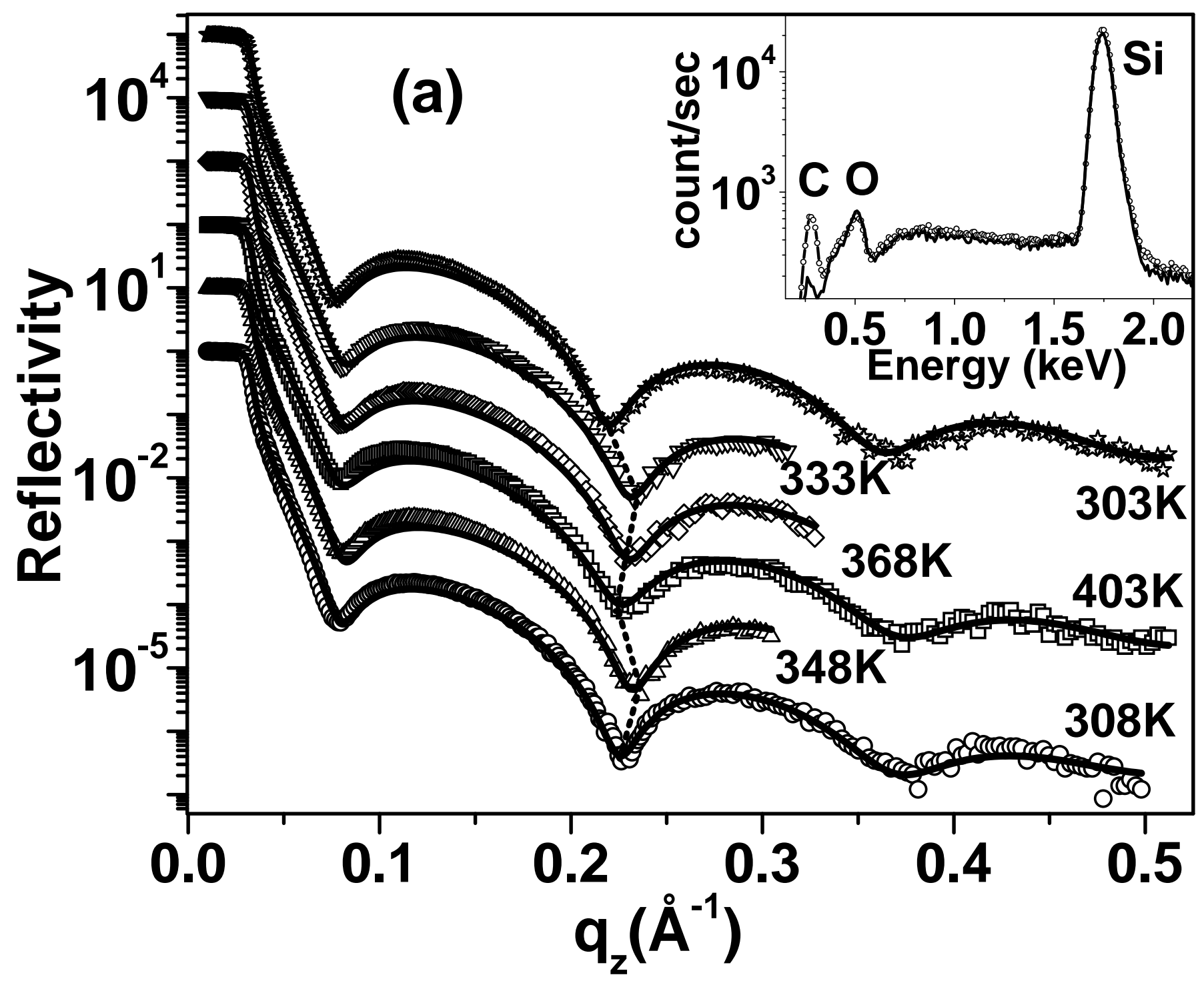




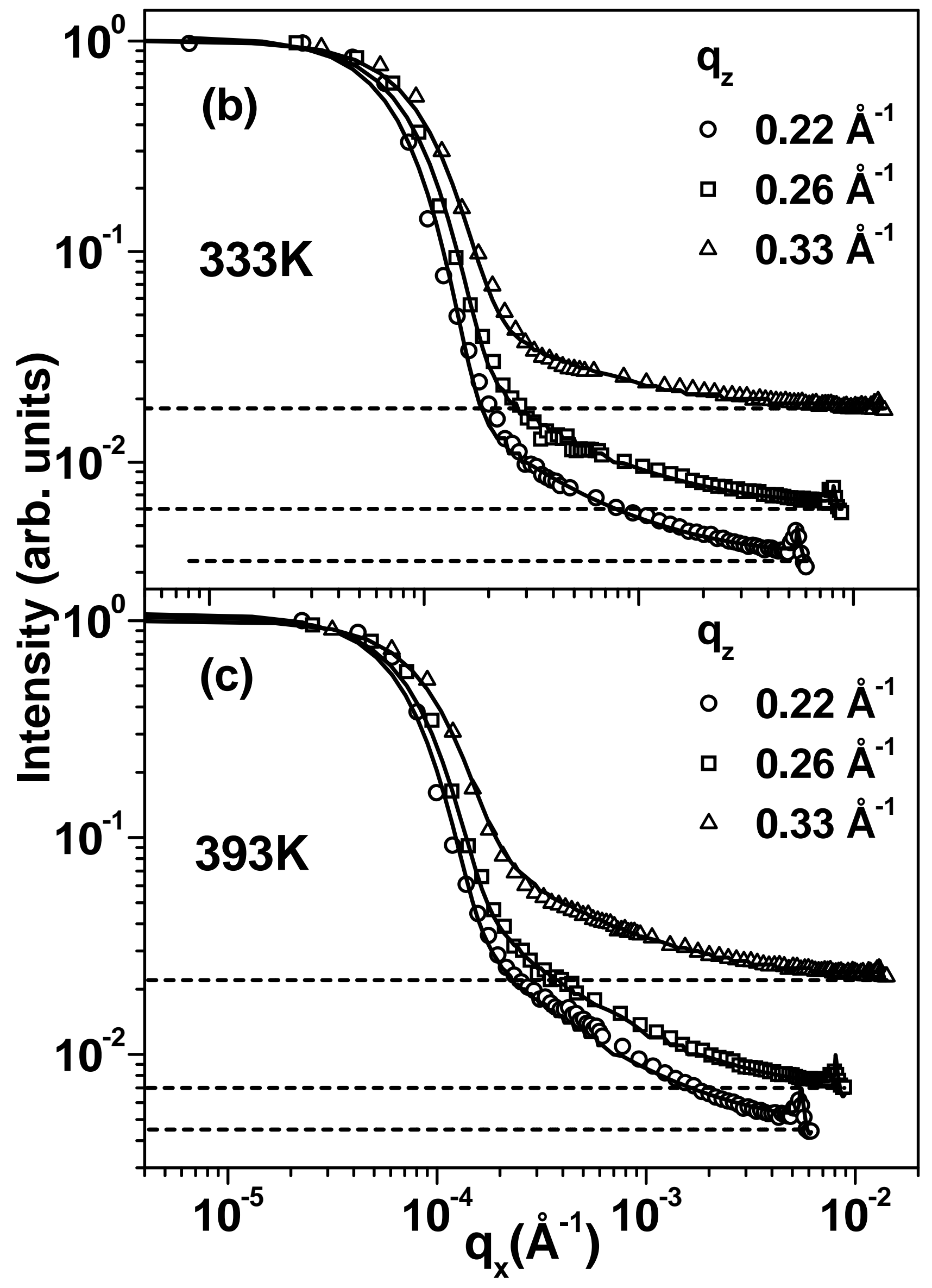




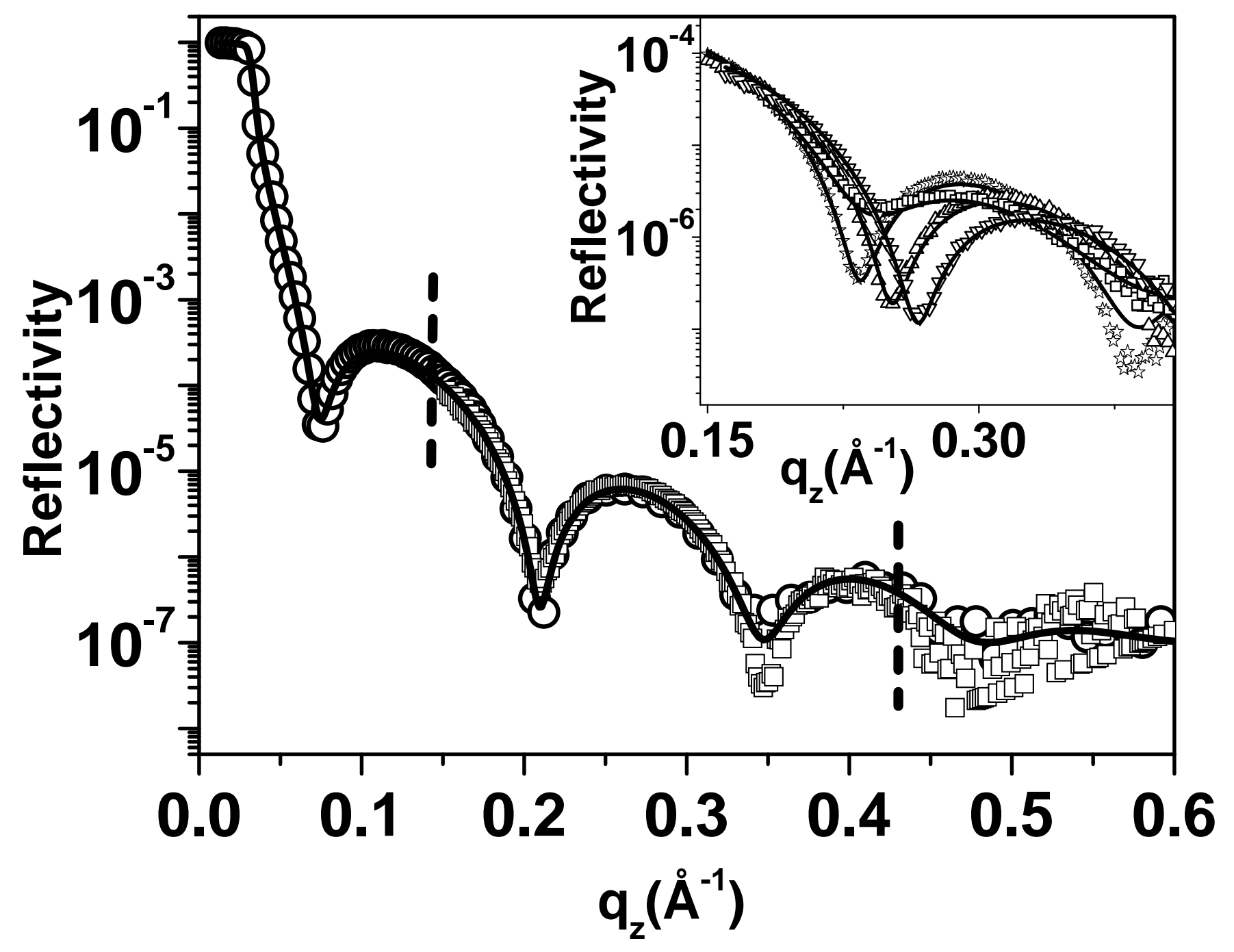




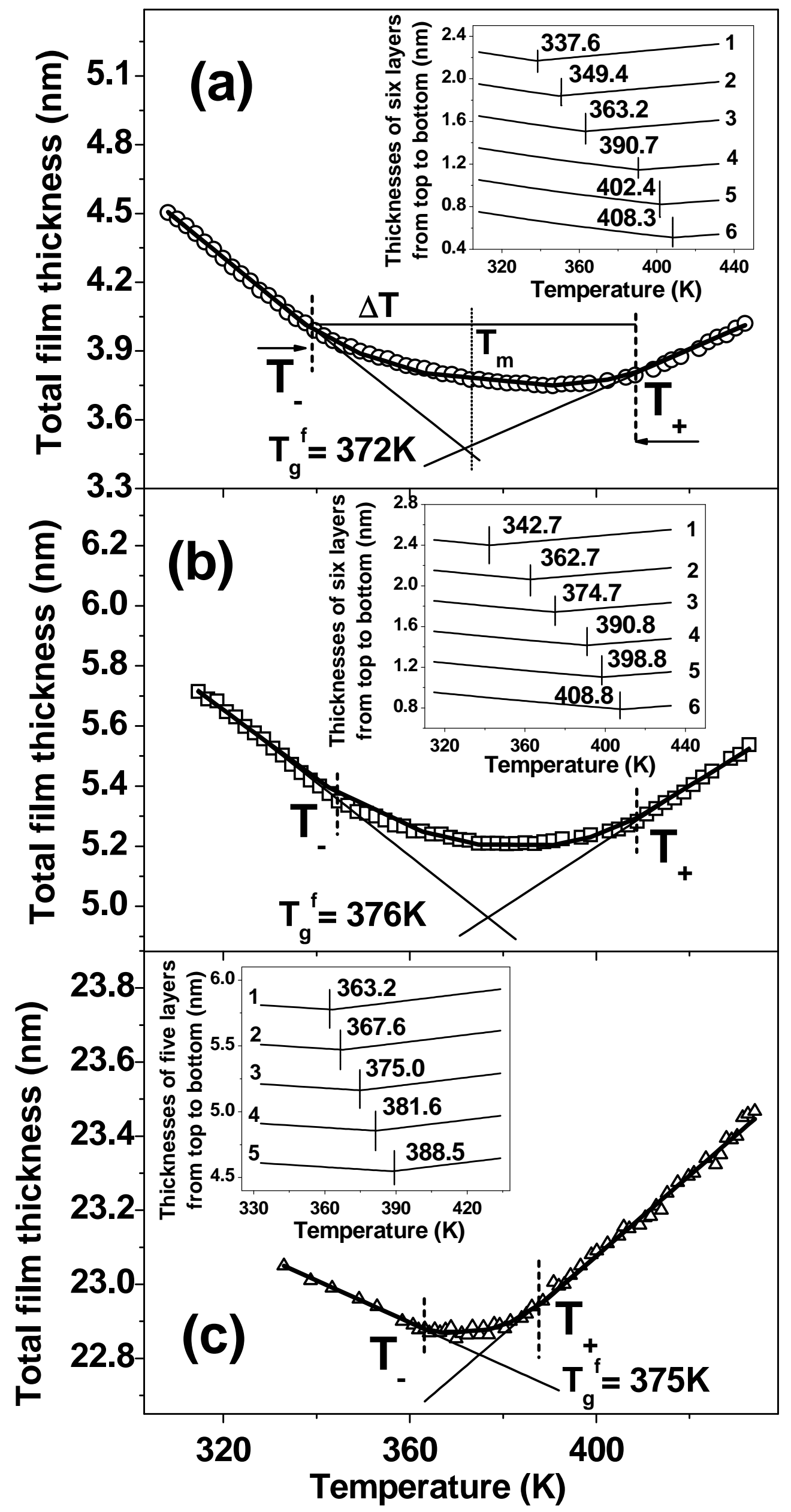




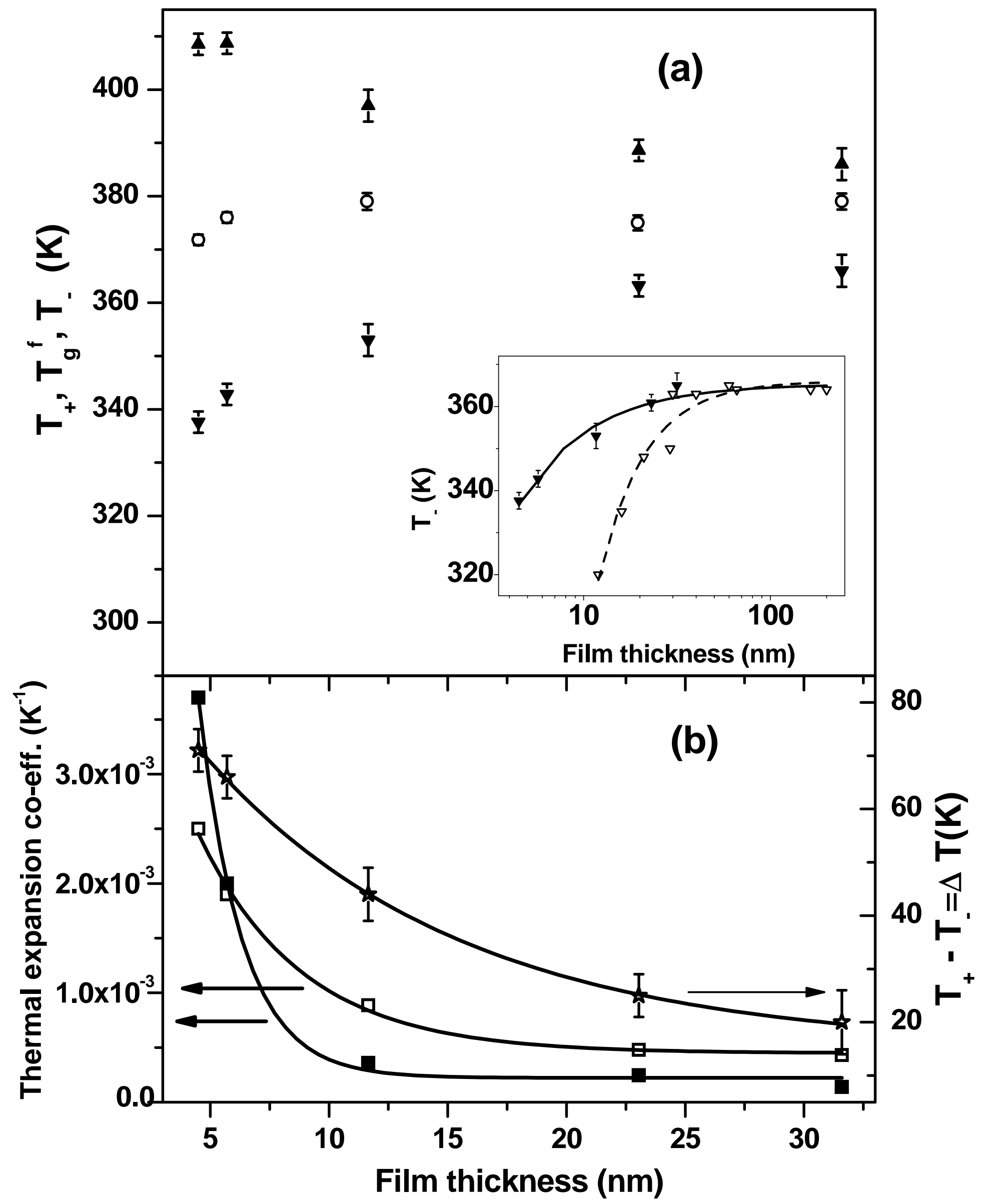

